\title{
Exploring Ground Level Ozone Distributions with Micromap Plots in Seoul of Korea
}

\author{
J. Y. Ahn and S. J. Park
}

\begin{abstract}
Increasing public concerns regarding elevated ozone levels and related health impacts have become an emerging issue in the world. In this study, we introduce two types of micromap plot, linked micromap plot and comparative micromap plot, to investigate the spatial and temporal trends of data and describe how spatial patterns of the data can be examined using the plots. To explore the potential for the use of the plots, we visualized ozone levels in Seoul of Korea for the period 1990-2013.
\end{abstract}

Index Terms - Data visualization, micromap plots, ozone levels, spatial patterns.

\section{INTRODUCTION}

Over the last two decades, Korea has experienced a rapid growth in urbanization, industrialization, and the number of motor vehicles. The resulting increased photochemical precursors have forced environmental policy-makers to recognize the potential urban ozone $\left(\mathrm{O}_{3}\right)$ problem [1].

Ozone is an omnipresent air pollutant that affects both human health and vegetation [2]. Increased public concern regarding elevated ozone levels and related health impacts have become an emerging issue in Korea as well [3]. Of particular concern is the nationwide prevalence of ozone problems burgeoning in Seoul, the capital of Korea, since many Korean cities are emulating Seoul as a model city in the various fields of societal systems. Since the first introduction in Seoul in 1995, the ozone warning systems continue to increase including neighbor cities of Seoul and other major cities.

Most high-ozone phenomena are characterized by local or provincial problems in Korea, which is different from current ozone problems in the eastern United States. In Korea, each province is relatively well isolated from neighboring provinces due to topographic features. Therefore, ozone problem has become the focus of increased national as well as local concern in Korea. As a result of the increased concern over ozone, the Korea Ministry of Environment established, alert, and serious alert levels for urban ozone concentrations along with appropriate accompanying measures.

In contrast to the increased national and local concern over ozone, only a very limited analysis of the characteristics of urban ground-level ozone has been conducted. Interpreting

Manuscript received November 5, 2014; revised May 20, 2015. This research was supported by the Basic Science Research Program through the National Research Foundation (NRF) of Korea funded by the Ministry of Education (NRF-2012R1A1A4A01002729).

The authors are with the Department of Statistics, Chonbuk National University, Jeonju, Jeonbuk, 561-756 Korea (e-mail: jyahn@jbnu.ac.kr, junesj@jbnu.ac.kr). and understanding air pollutant data usually requires considering an appropriate spatial context: most data come with geographic information, and many studies apply a geographical framework to analyze these data [4]-[6]. Most of these studies use simple images and maps, such as aerial images and choropleth and isopleth maps to visualize the data. Although these images and maps are commonly used, they also have problems and limitations [7]. One problem is that it is difficult to show more than one variable in an image or a map. Another problem is that it may be difficult and frustrating for a reader to observe trends, relationships, and anomalies that may be present in the data [8].

In this study, we introduce two types of micromap plot, linked micromap plot and comparative micromap plot, to investigate the spatial and temporal trends of data and describe how spatial patterns of the data can be examined using the plots. To explore the potential for the use of the micromap plots, we analyzed and visualized ozone levels in Seoul of Korea. With the plots, we can explore relationships between the variables and geographic patterns that are inherent in the data.

\section{DATA AND METHODS}

\section{A. Data Sources}

The Korean Regional Environmental Management Office and some municipal governments currently operate ozone monitoring stations in 117 urban and 2 remote sites. There are 25 sites in Seoul. Twelve readings per hour are typically used to calculate a 1-hour average concentration.

To analyze and visualize ozone and other pollutants data, we collected data for the period 1990-2013 from the KOSIS (Korean Statistical Information Service, http://kosis.kr), NIER (National Institute of Environmental Research, http://www.nier.go.kr), and SSIS (Seoul Statistical Information System, http://opengov.seoul.go.kr/stat). We aggregated the data into the 16 upper level local autonomies of Korea using the traditional geographic breakdown prior to the creation of Sejong as a special autonomous city in 2007. As of 2006, Korea was divided into eight provinces, one special autonomous province, six metropolitan cities, and one special city. In addition, we rearranged the data into the 25 districts of Seoul. Seoul is the business and financial hub of Korea. Although it accounts for only 0.6 percent of the nation's land area, it has a population of over 10 million and generates about 23 percent of the country's GDP. The data used in this study consists of 5 pollutants (ozone: $\mathrm{O}_{3}$, fine particle: $\mathrm{PM}_{10}$, sulphur dioxide: $\mathrm{SO}_{2}$, nitrogen dioxide: $\mathrm{NO}_{2}$, carbon monoxide: $\mathrm{CO}$ ) and region names. 


\section{B. Micromap Plots}

The graphical visualization techniques presented in this study are referred to as linked micromap (LM) plot and comparative micromap (CM) plot. LM plots are graphics that link statistical information to an organized set of small maps in order to explore and communicate patterns in the outcome variable, geographic locations and the associations among them [9].
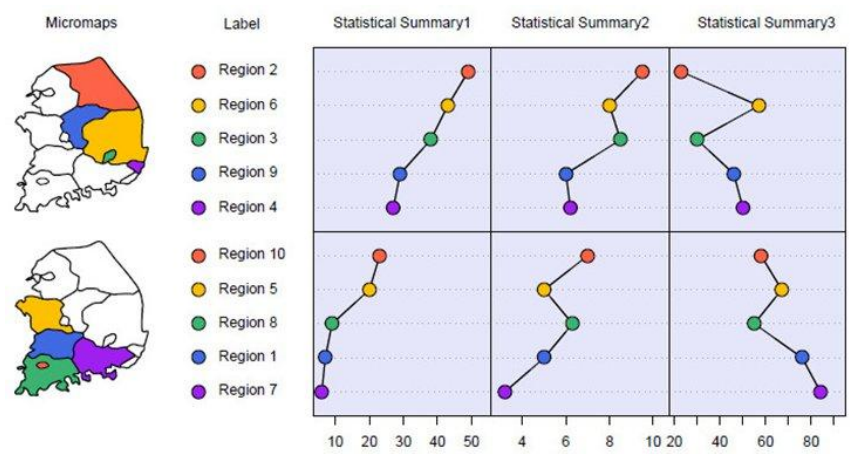

Fig. 1. A template of LM plot.

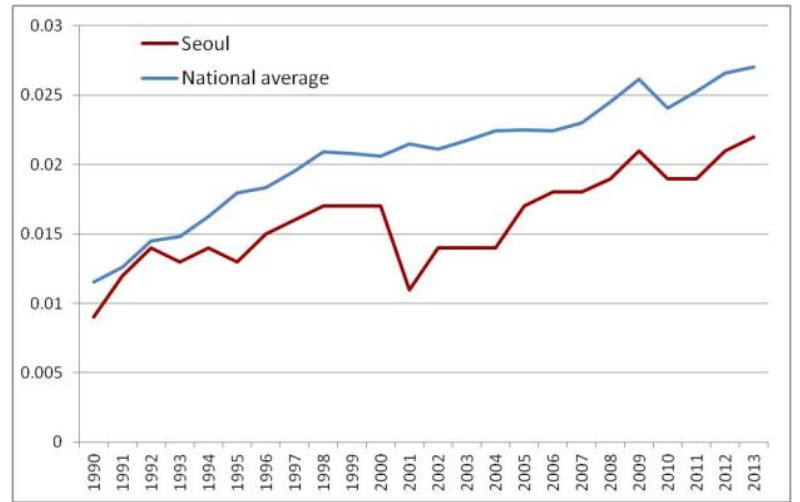

Fig. 2. Trends of ozone in Seoul and Korea from 1990 to 2013.
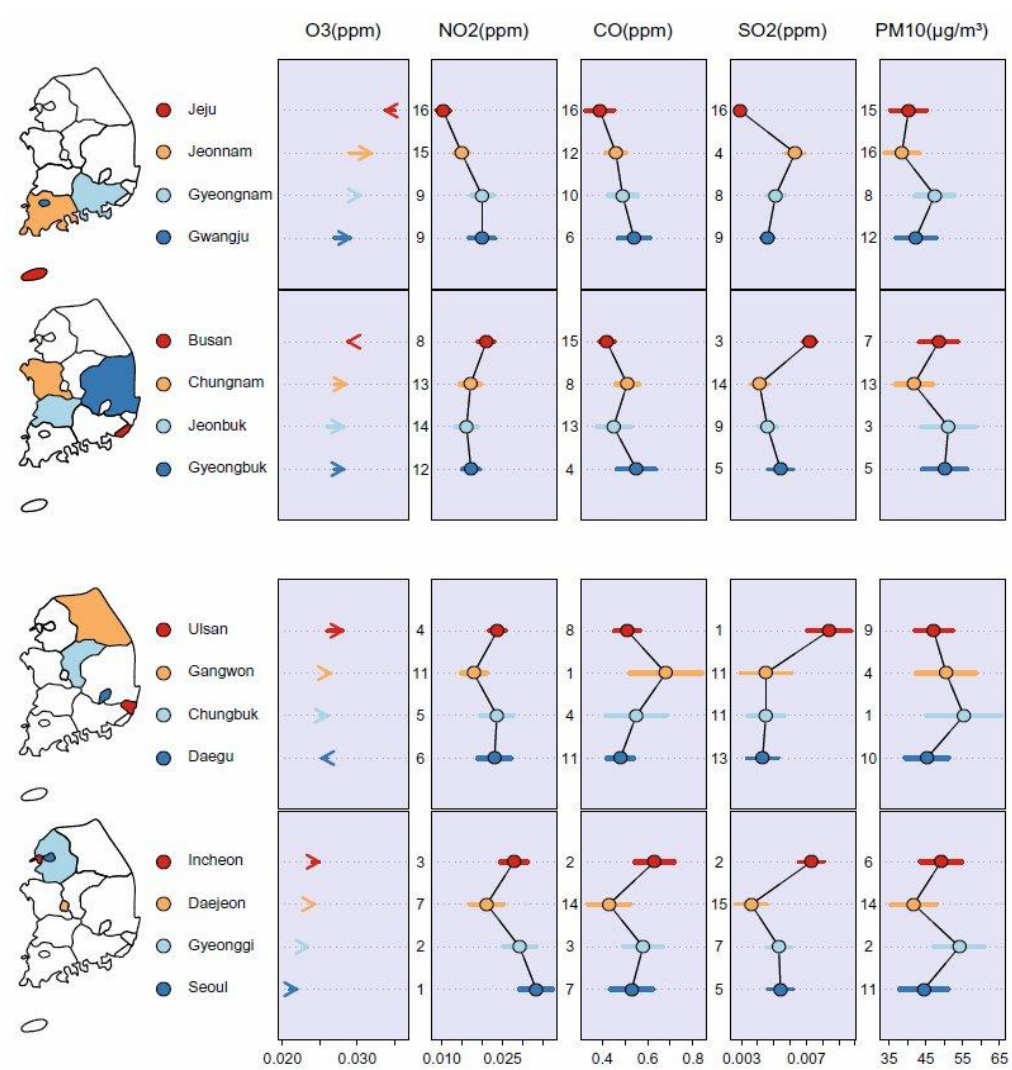

Fig. 4. LM plot showing spatial patterns of ozone levels and relationships between air pollutants for 2013 in Korea. lost with other types of graphs and maps.

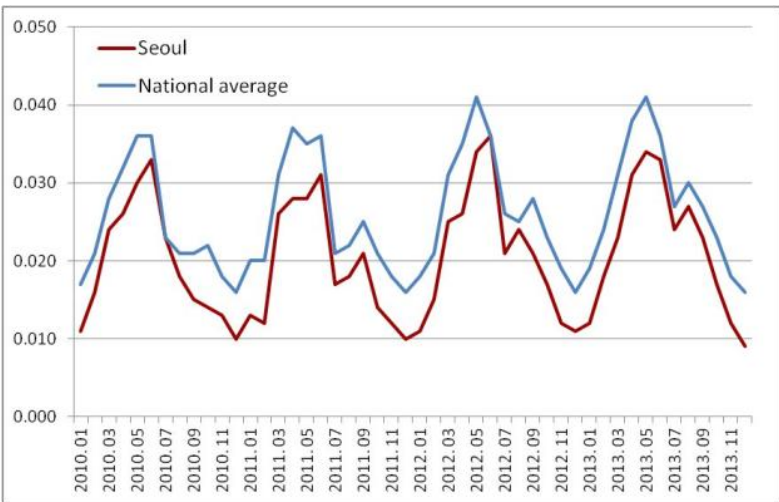

Fig. 3. Monthly trends of ozone.

LM plots are a collection of related plots presenting many statistical summaries from spatially referenced data in one graphical setting [10]. LM plots offer a new template for displaying spatially indexed statistical summaries. One can use LM plots to visualize complex data in many areas [11]. Fig. 1 presents a typical template for a LM plot. To visualize the geographically referenced data, LM plots use three or more sequence panels in parallel, linked by location. The types of panels are micromaps, labels, and statistical summary panels. Fig. 1 shows five parallel sequences of panels: one micromap, one label, and three statistical summary panels. LM plots can sort geographic subregions based on the statistical variables of interest. Sorting improves perception between consecutive panels, from the top to the bottom of the display. To allow the viewer to focus attention on explicit areas, one at a time, LM plots divide the regions into perceptual groups of size five or fewer, and link the locations using colors. These features of LM plots make it possible to visualize specific geographic patterns in the data that are often 


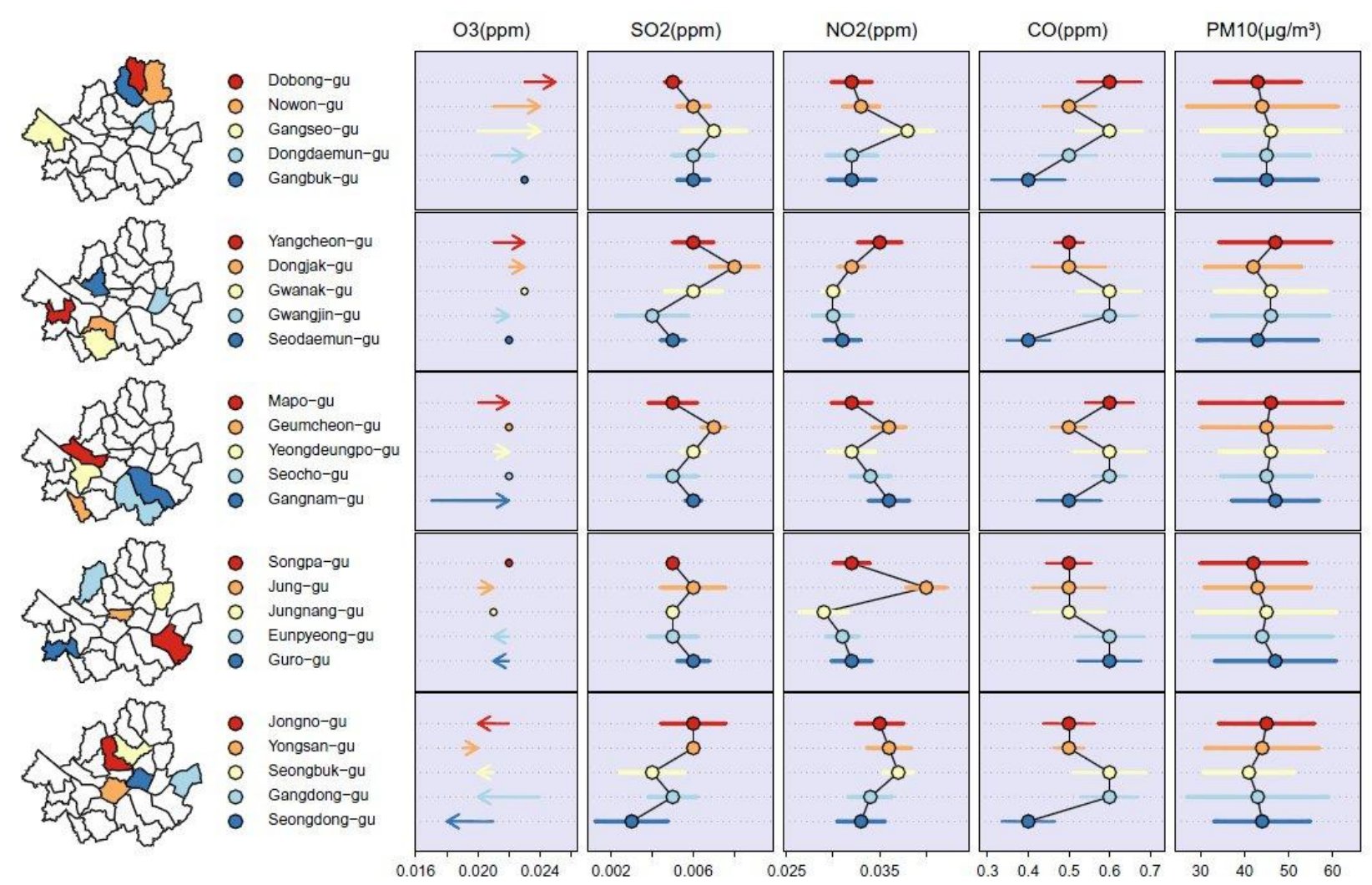

Fig. 5. LM plot showing spatial patterns of ozone levels and relationships between air pollutants for 2013 in Seoul.

CM plots are one-way and two-way sequences of organized small maps indexed by a categorical variable such as time, gender, age groups, and education levels. The primary purpose of CM plots is to emphasize spatial context, facilitate pattern discovery, and address change blindness [12]. The basic layout for a comparative micromap design is a row, column or matrix of complete micromaps, indexed by one or more factors. A typical application is the display of several maps in a row or column to show geographic changes over time. This common design expects the viewer to see the changes that occur from one map to the next. The comparative micromap design removes the cognitive burden of remembering multiple complex images and mentally subtracting values between maps, so that the reader can focus on examining the geographic patterns on the original maps or the difference maps [13].

\section{DATA EXPLORATION AND VISUALIZATION}

\section{A. Trends in Ozone}

The average levels of $\mathrm{PM}_{10}, \mathrm{SO}_{2}$, and $\mathrm{CO}$ in Korea fell to a record low last year thanks to the government's efforts to improve air quality. However, the level of $\mathrm{O}_{3}$ has been on the increase for the past twenty years.

Fig. 2 presents the temporal variations in ozone from 1990 to 2013 in Seoul and Korea. Though the level of ozone in Seoul is lower than national average level, the time series shows increasing trends. Fig. 3 shows the monthly variation of ozone from January 2010 to December 2013. The levels of ozone exhibited distinct seasonal changes: the levels were higher from February to April and then lower from November to January. Ozone measurements were highest in May, when levels were more than three times those in December.

\section{B. Data Visualization Using Micromap Plots}

LM plots are graphics that link statistical information to an organized set of small maps to explore and communicate patterns in the outcome variable, geographic locations, and the associations among them [14].

Fig. 4 shows how an LM plot can illustrate spatial patterns of ozone levels and relationships between air pollutants. The data shown are averages for 2013, and regions in the figure are sorted by ozone levels, from highest to lowest. The map panel of the LM plot exhibits a geographic pattern: highest level of ozone (shown in the statistical panel 1) can be found in the south; lowest level can be found in the west and in the north. Ozone does not show any association with $\mathrm{CO}, \mathrm{SO}_{2}$ and $\mathrm{PM}_{10}$ (Spearman's rho: $-0.389,-0.068$ and -0.465 , p-values: $0.137,0.803$ and 0.070 , respectively), but ozone and $\mathrm{NO}_{2}$ have a negative association (Spearman's rho: -0.809 , p-value: 0.000). In addition, $\mathrm{CO}$ and $\mathrm{PM}_{10}$ are positive correlated (Spearman's rho: 0.563, p-value: 0.023). The levels of ozone in 2013 compared with 2012 have increased in all local autonomies.

Fig. 5 presents the levels of ozone and other pollutants in Seoul, the capital city of Korea. The highest level of ozone can be found in the north; lowest level can be found in the central part. In Seoul, ozone and $\mathrm{SO}_{2}$ have a positive association (Spearman's rho: 0.430, p-value: 0.032), but ozone does not show any association with other pollutants. In addition, the arrows in ozone of the figure indicate increasing levels in 2013 compared with 2012. The greatest increase region in ozone level compared with 2012 is Gangnam-gu; the greatest decrease region is Gangdong-gu.

Fig. 6 is an example of CM plot to map the ozone levels for Seoul from 1998 to 2013. CM plot is a useful tool for 
visualizing changes in geographic data. The highest $25 \%$ of the levels for the years were shaded red, the lowest $25 \%$ were shaded blue, and the rest were shaded gray. In the second row, each map has shaded only those provinces that changed categories from one year to next in the first row. The second and third rows (the first and second maps of differences) show pairwise differences between maps in order to use most basic cognitive process to judge patterns; i.e., seeing explicit differences is quicker than calculating them. There were more high-level districts (red) in 2010 and 2013, with gradually high levels on all regions. In 2001 and 2004, it has the most low-level regions, but most regions changed to the medium and high category in 2010. Only one region shows the low category in 2010, and there was a deterioration since 2004.

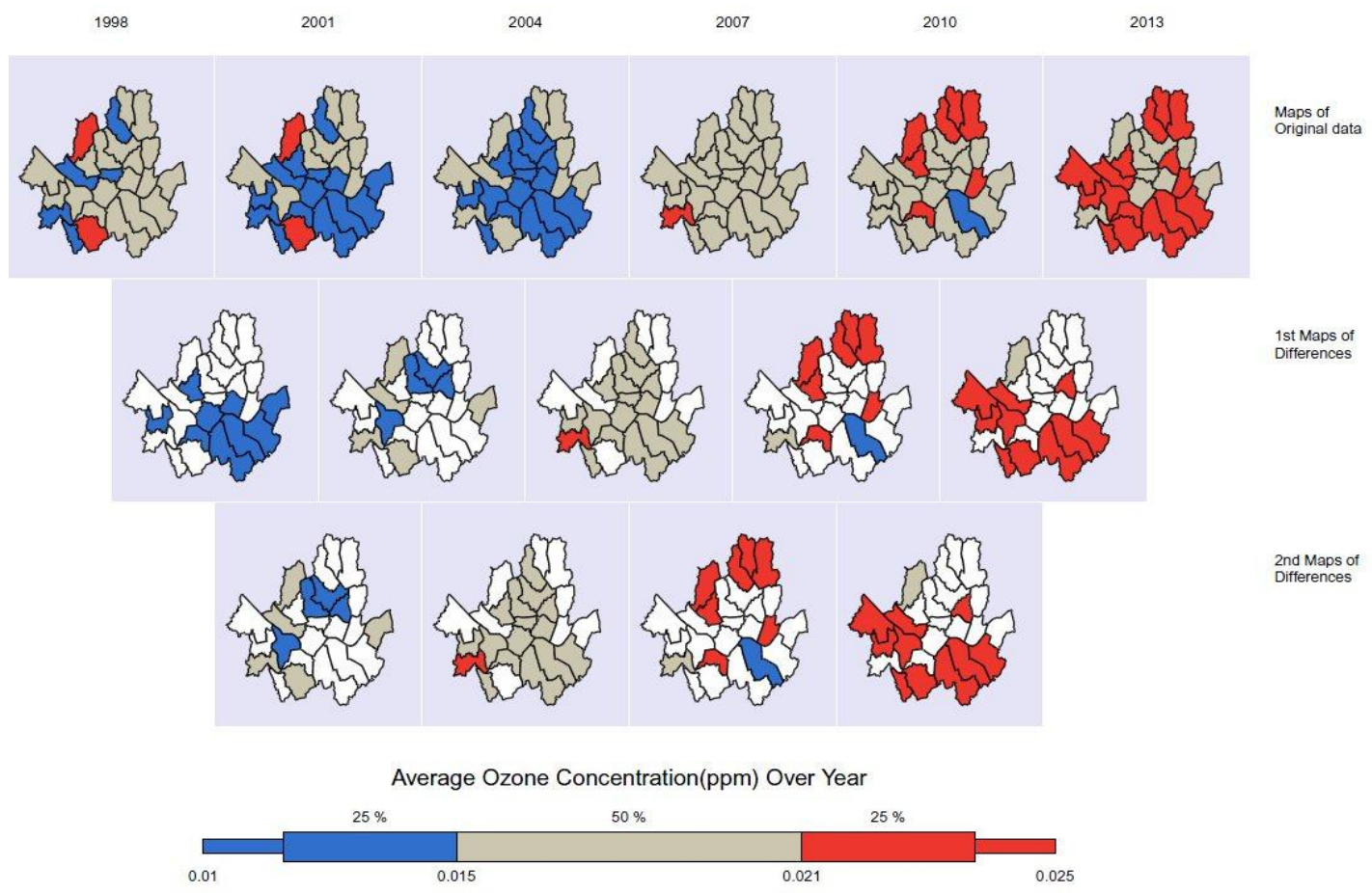

Fig. 6. CM plot of annual ozone levels from 1998 to 2013 in Seoul.

\section{CONCLUSION}

Maps have the potential to display the geographic patterns of millions of statistical data points, something that is impossible using a tabular display. In this study, we introduce two types of micromap plot, linked and comparative micromap plot, for visualizing statistical information of geographically referenced data. We examined the trends of air pollution for the period from 1990 to 2013 in Korea and Seoul and demonstrated how spatial patterns of the air pollutants can be examined simultaneously using LM plots.

First, we investigated temporal variations in pollutants at national and local (Seoul) level. Second, we explored relationships between the pollutants and geographic patterns/trends of ozone levels using LM and CM plots. With the micromap plots, we can present and visualize more enriched statistical information, help readers to better understand the variety of statistical data, and explore the geographic patterns that inhere in the data.

There are several remaining problems for future exploration. First, we should consider more types of plots for generalization, even if the types considered in this study are the most common. It also requires to extend the function for micromap plots to control the position of panels.

\section{REFERENCES}

[1] W. K. Jo and C. W. Nam, "Characteristics of urban ground-level ozone in Korea," Journal of the Air \& Waste Management Association, vol. 49, pp. 1425-1433, 1999.
[2] A. S. Lefohn and J. K. Foley, "Establishing relevant ozone standards to protect vegetation and human health: Exposure/dose-response considerations," Journal of the Air \& Waste Management Association, vol. 43, pp. 106-112, 1993.

[3] S. H. Cho, Y. S. Joo, J. H. Sung, J. W. Kang, C. Y. Kim, S. W. Choi, and H. J. Kwon, "Health effects of ambient ozone in Seoul (in Korean)," in Proc. 1998 KAPRA Fall Meeting, 1998.

[4] L. Matejicek, "Spatial modelling of air pollution in urban areas with GIS: A case study on integrated database development," Advances in Geosciences, vol. 4, pp. 63-68, 2005.

[5] M. Jerrett, R. T. Burnett, R. Ma, C. A. Pope, D. Krewski, K. B. Newbold, G. Thurston, Y. Shi, N. Finkelstein, E. Calle, and M. J. Thun, "Spatial analysis of air pollution and mortality in Los Angeles," Epidemiology, vol. 16, pp. 727-736, 2005.

[6] O. Eitana, O. Yuval, M. Barchana, J. Dubnov, S. Linnd, Y. Carmel, and D. M. Broday, "Spatial analysis of air pollution and cancer incidence rates in Haifa Bay, Israel," Science of the Total Environment, vol. 408, pp. 4429-4439, 2010.

[7] R. L. Harris, Information Graphics - A Comprehensive Illustrated Reference, NY: Oxford University Press, 1999.

[8] S. Gebreab, R. R. Gillies, R. G. Munger, and J. Symanzik, "Visualization and interpretation of birth defects data using linked micromap plots," Birth Defects Research (Part A), vol. 82, pp. 110-119, 2008.

[9] D. B. Carr and S. M. Pierson, "Emphasizing statistical summaries and showing spatial context with micromaps," Statistical Computing and Graphics Newsletter, vol. 7, pp. 16-23, 1996.

[10] L. A. Waller and C. A. Gotway, Applied Spatial Statistics for Public Health Data, NY: John Wiley \& Sons, 2004.

[11] X. Wang, J. X. Chen, D. B. Carr, B. S. Bell and L. W. Pickle, "Geographical statistics visualization: Web-based linked micromap plots," Computing in Science \& Engineering, vol. 4, pp. 90-94, 2002.

[12] C. Zhang, "Interfaces and visual analytics for visualizing spatio-temporal data with micromaps," Ph.D. dissertation, George Mason University, Fairfax, VA, 2012.

[13] L. W. Pickle and D. B. Carr, "Visualizing health data with micromaps," Spatial and Spatio-Temporal Epidemiology, vol. 1, pp. 143-150, 2010. 
[14] D. B. Carr and L. W. Pickle, "visualizing data patterns with micromaps," FL: Chapman and Hall/CRC, 2010.

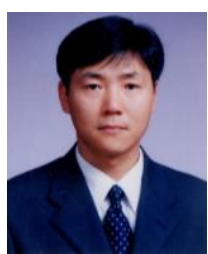

J. Y. Ahn was born in South Korea. He received the B.S., M.S., and Ph.D. degrees from Chonbuk National University, Jeonbuk, Korea, in 1992, 1994, and 2001, respectively. He was a faculty of the Department of Computer Science and Statistics, Seonam University, from 1995. In 2003, he joined the Faculty of the Department of Statistics, Chonbuk National University, where he is currently a professor. His research interests include statistical maps, e-learning, data mining and database applications.

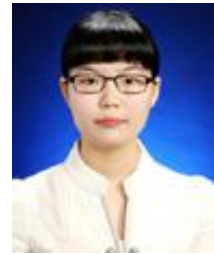

S. J. Park was born in South Korea. She received the B.S. and M.S. degrees from Chonbuk National University, Jeonbuk, Korea, in 2011 and 2013, respectively. She is pursuing his doctoral degree at the Department of Statistics, Chonbuk National University, Korea. Her research interests include statistical maps, data visualization and data mining. 